\title{
Comparison and reproducibility of standard and high temporal resolution myocardial tissue tagging in patients with severe aortic stenosis
}

\author{
Christopher D Steadman ${ }^{1 *}$, Naveed A Razvi ${ }^{1}$, Kym IE Snell ${ }^{2}$, Joost PA Kuijer ${ }^{3}$, Albert C van Rossum ${ }^{4}$ \\ Gerry P McCann ${ }^{2}$
}

From 2011 SCMR/Euro CMR Joint Scientific Sessions

Nice, France. 3-6 February 2011

\section{Objectives}

The aim of this study was to compare and assess the reproducibility of left ventricular (LV) circumferential peak systolic strain $\left(\right.$ PeakE $\left._{\mathrm{cc}}\right)$ and strain rate $(\mathrm{SR})$ measurements using standard and high temporal resolution myocardial tissue tagging in patients with severe aortic stenosis (AS).

\section{Background}

Myocardial tissue tagging with cardiac magnetic resonance (CMR) can be used to quantify strain and SR, however, there are little data on the reproducibility. Diastolic SR may be of particular interest as it may be the most sensitive marker of diastolic dysfunction often occurring early in the course of disease.

\section{Methods}

Eight patients with isolated severe AS without obstructive coronary artery disease were prospectively enrolled. They underwent CMR in a $1.5 \mathrm{~T}$ scanner (Siemens Avanto) on two separate occasions, median interval 12 days. Complementary tagged (CSPAMM) images were acquired with both a single breath-hold (SBH: temporal resolution $42 \mathrm{~ms}$ ), and a multiple brief expiration breath-hold (MBH: high temporal resolution $17 \mathrm{~ms}$ ) sequence. Mid-wall PeakE $\mathrm{c}_{\mathrm{cc}}$ was measured in the LV at mid-ventricular level with HARP Version 2.7 (Diagnosoft, USA). SR was calculated from the strain data; $\mathrm{SR}=\mathrm{E}_{\mathrm{cc}} 2-\mathrm{E}_{\mathrm{cc}} 1 /$ Time2-Time1. PeakE $\mathrm{E}_{\mathrm{cc}}$, peak systolic and diastolic SR were read from curves of strain and SR against time. The MBH SR curves were filtered with a

Table 1 Results

\begin{tabular}{|c|c|c|c|c|c|}
\hline & & $\begin{array}{l}\text { Peak systolic strain } \\
(\%)\end{array}$ & $\begin{array}{l}\text { Peak systolic strain } \\
\text { rate }(1 / s)\end{array}$ & $\begin{array}{l}\text { Peak diastolic strain } \\
\text { rate }(1 / s)\end{array}$ & \\
\hline $\mathrm{SBH}$ & & $-13.7 \hat{A} \pm 2.4$ & $-0.74 \hat{A} \pm 0.15$ & $0.75 \hat{A} \pm 0.27$ & \\
\hline MBH (MA of three) & & $\begin{array}{l}-15.1 \hat{A} \pm 3.1 \quad(p=0.023 \\
\text { vs. } S B H)\end{array}$ & $\begin{array}{l}-0.73 \hat{A} \pm 0.11(p=0.877 \\
\text { vs. SBH) }\end{array}$ & $\begin{array}{l}1.12 \hat{A} \pm 0.54(p=0.017 \text { vs. } \\
S B H)\end{array}$ & \\
\hline MBH (MA of five) & $\begin{array}{l}-0.69 \hat{A} \pm 0.10(p=0.049 \\
\text { vs. } S B H)\end{array}$ & & & $\begin{array}{l}0.91 \hat{A} \pm 0.36(p=0.535 \text { vs. } \\
S B H)\end{array}$ & \\
\hline SBH reproducibility & MDÂASD CoV B-A & & $\begin{array}{l}0.50 \hat{A} \pm 1.5211 .1 \%-2.5 \\
\text { to } 3.5\end{array}$ & $\begin{array}{l}-0.01 \hat{\mathrm{A}} \pm 0.1318 .1 \%-0.26 \\
\text { to } 0.28\end{array}$ & $\begin{array}{l}-0.04 \hat{\mathrm{A}} \pm 0.1621 .0 \%-0.36 \\
\text { to } 0.27\end{array}$ \\
\hline $\begin{array}{l}\text { MBH reproducibility } \\
\text { (MA of three) }\end{array}$ & MDÂASD CoV B-A & $\begin{array}{l}1.13 \hat{\mathrm{A}} \pm 2.2314 .7 \% \\
-3.3 \text { to } 5.6\end{array}$ & $\begin{array}{l}0.06 \hat{\mathrm{A}} \pm 0.045 .3 \%-0.02 \\
\text { to } 0.14\end{array}$ & $\begin{array}{l}-0.13 \hat{A} \pm 0.4439 .0 \%-1.00 \\
\text { to } 0.75\end{array}$ & \\
\hline $\begin{array}{l}\text { MBH reproducibility } \\
\text { (MA of five) }\end{array}$ & MDÂASD CoV B-A & & $\begin{array}{l}0.04 \hat{A} \pm 0.057 .8 \%-0.07 \\
\text { to } 0.15\end{array}$ & $\begin{array}{l}0.09 \hat{\mathrm{A}} \pm 0.1516 .9 \%-0.39 \\
\text { to } 0.22\end{array}$ & \\
\hline
\end{tabular}

$\mathrm{MD} \pm \mathrm{SD}=$ mean difference \pm standard deviation, $\mathrm{CoV}=$ coefficient of variation, $\mathrm{B}-\mathrm{A}=\mathrm{Bland}-\mathrm{Altman} 95 \%$ limits of agreement.

'Department of Cardiovascular Sciences, University of Leicester, Leicester, UK

Full list of author information is available at the end of the article 

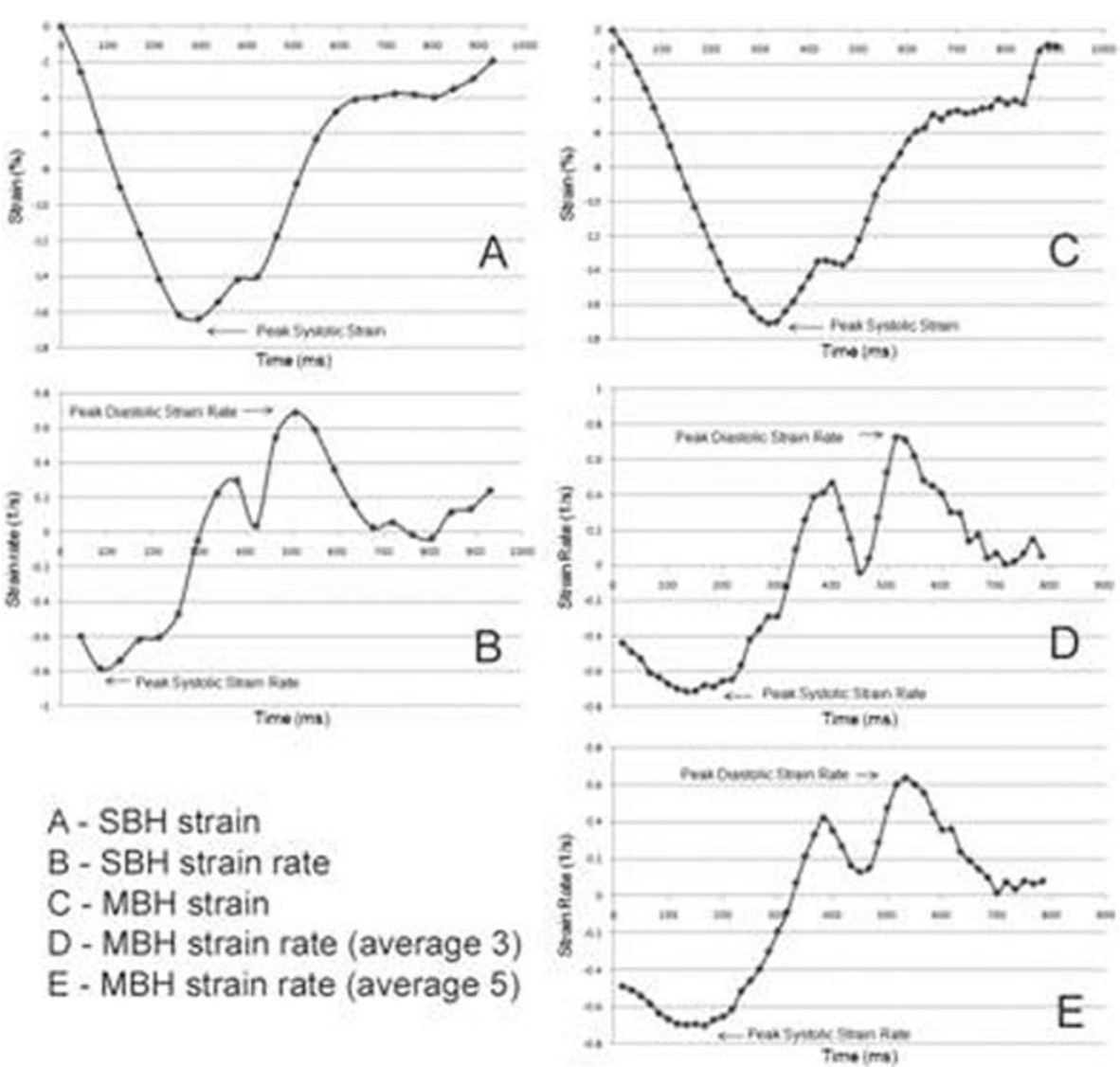

Figure 1 Strain and SR curves.

moving average (MA) to reduce noise sensitivity, results from a sample width of three and five were examined. Differences between SBH and MBH were assessed using Wilcoxon signed-rank test as not all measures were normally distributed. Reproducibility assessments were carried out on all techniques.

\section{Results}

PeakE $\mathrm{cc}_{\mathrm{cc}}$ was significantly higher with $\mathrm{MBH}$ vs. $\mathrm{SBH}$, but reproducibility was slightly worse. Results are summarised in Table 1. Systolic SR was approximately equal with all techniques although MBH using MA of five led to a borderline significant reduction. Diastolic SR was higher when measured with MBH although only significant using MA of three. Systolic and diastolic SR measures were more reproducible with $\mathrm{MBH}$ compared with $\mathrm{SBH}$, except for the diastolic SR using MA of three, which was substantially worse. Strain and SR curves for the same patient are shown in Figure 1.

\section{Conclusions}

It is likely than $\mathrm{SBH}$ may be adequate or even superior to $\mathrm{MBH}$ for assessment of PeakE $\mathrm{cc}_{\mathrm{cc}}$. The increased temporal resolution of $\mathrm{MBH}$ may be advantageous for examining systolic and diastolic SR; a MA of five for diastolic SR may be the preferred method for quantification given the improved reproducibility of this measure.

\section{Author details}

${ }^{1}$ Department of Cardiovascular Sciences, University of Leicester, Leicester, UK ${ }^{2}$ NIHR Leicester Cardiovascular Biomedical Research Unit, Leicester, UK. ${ }^{3}$ Department of Physics and Medical Technology, ICaR-VU, VU University Medical Center, Amsterdam, Netherlands. ${ }^{4}$ Department of Cardiology, ICaRVU, VU University Medical Center, Amsterdam, Netherlands.

Published: 2 February 2011

doi:10.1186/1532-429X-13-S1-P311

Cite this article as: Steadman et al.: Comparison and reproducibility of standard and high temporal resolution myocardial tissue tagging in patients with severe aortic stenosis. Journal of Cardiovascular Magnetic Resonance 2011 13(Suppl 1):P311. 